Bangladesh J. Plant Taxon. 28(1): 141-154, 2021 (June)

(C) 2021 Bangladesh Association of Plant Taxonomists

https://doi.org/10.3329/bjpt.v28i1.54214

\title{
EVALUATION OF PLANT SPECIES DIVERSITY IN THE ROAD DIVIDERS OF DHAKA CITY
}

\author{
Mohammad Zashim Uddin ${ }^{1}$, Abulais Shomrat, Md. SiddiQ Hasan, \\ Md. Robin Khan, Abdur Rahman Fahad and Md. Al-Amin \\ Department of Botany, University of Dhaka, Dhaka-1000, Bangladesh
}

Keywords: Evaluation, Dominance, Road dividers, Plant species diversity, Dhaka city.

\begin{abstract}
The present study aims to evaluate the present status of plant species diversity in the road dividers of Dhaka city. A total of 90 plant species under 73 genera and 42 families have been recorded from the road dividers of Dhaka city. Analysis showed that among the 90 species, the most abundant plant species in the road divider are Mimusops elengi (Bakul) Polyalthia longifolia (Debdaru), Leucaena leucocephala (Epil-epil), Tabernaemontana divaricata (Togor) and Swietenia mahagoni (Mahagoni). In case of dominant analysis based important value index showed that Mimusops elengi is the top dominant plant species followed by Polyalthialongifolia, Leucaena leucocephala, Tabernaemontana divaricata and Swietenia mahagoni respectively. These plant species have also showed the higher relative density, relative frequency and relative abundance. The percentage of native and exotic species was found here $44 \%$ and $56 \%$, respectively. Most abundant and dominant plant species in the road dividers are exotics including Mimusops elengi,Polyalthia longifolia, Leucaena leucocephala and Swietenia mahagoni. Species diversity value was found higher in Hatirjeel site whereas lowest in BaridharaBadda site. A number of recommendations are made based on the present results for the management of plant species diversity in the road dividers of Dhaka city. Some recommended native plant species for plantation according to the width and soil depth in the road dividers are Cassia fistula, Holarrhena pubescens, Firmiana colorata, Shorea robusta, Miliusa valutina, Butea monosperma, Alstonia scholaris, Toona ciliata, Ficus racemosa, Bombax ceiba, Woodfordia fruticosa, Melastoma malabathricum, Sterculia villosa, Grewia microcos, Syzygium cumini, Tabernaemontana divaricata, Ixora coccinea, Terminalia bellirica, Terminalia arjuna and also native seasonal flowers.
\end{abstract}

\section{Introduction}

Plant species diversity of urban areas has received considerable attention in the last few decades (Sukopp, 1990) and urban green spaces are being considered critical for biodiversity conservation (Aronson et al., 2017). It was observed that, in floristic terms, the floral diversity of urban areas is richer when compared to the suburbs due to higher habitat heterogeneity and the presence of exotic species (Pyšek, 1998). Urbanization facilitates the expansion of the introduced species (McKinney, 2008; Pyšek and Richardson, 2010). Sometimes urbanization destroys natural habitats and displaces native biota (Smith et al., 2005; Marco et al., 2008). As urban areas provide an important depositories of plant resource which has huge benefits for humans and as well as animals, plant diversity in urban areas, particularly in the tropics, needs to be assessed and conserved (Singh et al., 2018).

A number of research works on plant species diversity of urban areas of Dhaka city were conducted. The noteworthy works are Datta and Mitra (1953); Alam (1967); Hossain (1966); Hossain (2006); Huq and Begum (1984); Hussain (1965); Khan and Huq (1981); Rahman (1966);

\footnotetext{
${ }^{1}$ Corresponding author: zashim01@ gmail.com
} 
Zeauddin (1967); Hossain and Uddin (2011); Uddin and Hassan (2016) and Uddin et al.(2019). All these works were covered the list of plants species or list of plant species of particular families or groups. Unfortunately, no works covered the abundance of urban plant species diversity of road dividers in Dhaka city. As the city expands, various types of trees are being planted on the road dividers every year. Over the past few years, those trees have become a source of grief for the people of city in some cases (The Daily Star May 11, 2018; March 8, 2016; March 19, 2017). Preliminary observations proved that in most cases biodiversity promotion and native plants conservation issues ignored to some extend or plant taxonomist knowledge was not taken much into account when planting these tree species. Now is the time to redecorate our favorite Dhaka city with native tree species based on scientific data. For this, it is necessary to know what kinds of trees are currently covered by road dividers in Dhaka city. In the present study, attempt has been made with the following objectives: to make an inventory of plant species diversity in the road dividers of Dhaka city; to determine the dominant plant species in the road dividers; to confirm whether the dominant species are native or exotics; to measure plant species diversity; to make recommendations for better management of plant diversity in Dhaka city.

\section{Materials and Methods}

Study area

Dhaka is the capital city of Bangladesh and growing ever since its independence. Geographically, the city is located in between latitudes $23^{\circ} 42^{\prime}$ and $23^{\circ} 54^{\prime} \mathrm{N}$ and longitudes $90^{\circ} 20^{\prime}$ and $90^{\circ} 28^{\prime} \mathrm{E}$ (Sayed et al. 2015). As a part of the Bengal plain, this city is bordered in the periphery by the Buriganga river in the south; the Balu and the Shitalakhya rivers in the east; Tongi canal in the north and the west is bounded by Turag and Buriganga rivers (Banglapedia 2014).Believed that once Dhaka city was the extend part of natural Sal (Shorea robusta) forest of Bhawalgarh and also with many water bodies. Most of the natural vegetation and water bodies of Dhaka city have been degraded by rapid urbanization and development activities. Currently, no natural forests present in Dhaka city. Major plantations in the road dividers, footpaths, city parks have been done by different stakeholders of Dhaka city including City Corporation, RAJUK and Public Work Department. Lawns of houses were planted by land owners in some cases. Remote sensing study proved that about 20\% green cover that was present in Dhaka city in 1989 has been gradually decreased to $15.5 \%$ and $7.3 \%$ in 2002 and 2010 respectively (Rahman et al. 2011). The city of Dhaka experiences a hot, wet and humid tropical climate. The city has a distinct monsoonal season, with an annual average temperature of $27.5{ }^{\circ} \mathrm{C}$. The city experiences about $2000 \mathrm{~mm}$ annual rainfall, of which more than $80 \%$ occurs during the monsoon season (June-September) (Dewan and Yamaguchi, 2009). Besides tropical nature of vegetation and moist soils characterize the land, which is flat and close to sea level leaving Dhaka susceptible to flooding during the monsoon seasons owing to heavy rainfall and cyclones (Hough 2004).

\section{Methods}

For the plant diversity data collection, quadrates of $5 \mathrm{mx} 5 \mathrm{~m}$ were placed at randomly selected sites (Table 1) on the road dividers in different areas of Dhaka city (Krebs, 1989). A total of 452 quadrates were surveyed. The number of quadrates in study area was determined using species area curve (Goldsmith and Harrison, 1976). Plant species with the number of individuals within the selected quadrate was recorded. Maximum identification of plant species was done in field by the expert consultation. In case of confusion, images of plants were taken and later were identified by comparing with standard literature (Siddiqui et al., 2007; Ahmed et al., 2008a,b; Ahmed et al. 2009 a,b,c,d) and by also comparing with the herbarium specimens available at Dhaka University Salar Khan Herbarium (DUSH). Some exotic plant species were determined comparing with the 
reports of Pasha and Hossain (2004) and Akter and Zuberi (2009). The families have been determined according to the classification system of Cronquist (1981). To determine dominant plant species in Dhaka city, Importance Value Index was calculated using biostatistical formulas (Krebs 1989). Species diversity was determined using Shannon-Weiner diversity index (Shannon, 1948) and Margalefindex (Margalef, 1957).

Table 1. Roads and name of places where transect lines have been placed.

\begin{tabular}{|c|c|c|}
\hline Site & Name of Places & Roads \\
\hline 1 & Uttara & $\begin{array}{l}\text { Jashimuddin Avenue } \\
\text { Road no. } 18 \\
\text { Rabindra Sarani }\end{array}$ \\
\hline 2 & Mirpur & $\begin{array}{l}\text { Darus Salam Road } \\
\text { Mirpur Road (up to Mirpur 14) }\end{array}$ \\
\hline 3 & Hatirpul- Panthapath & $\begin{array}{l}\text { Katabon Road } \\
\text { Sonargaon Road } \\
\text { Bir Uttam Kazi Nuruzzaman Road }\end{array}$ \\
\hline 4 & $\begin{array}{l}\text { Dhanmondi- Parliament } \\
\text { Area- Azimpur }\end{array}$ & $\begin{array}{l}\text { Mirpur Road (From Nilkhet to Aarong Lalamatia) } \\
\text { Manik Mia Avenue } \\
\text { Road no. } 27 \\
\text { Satmasjid Road } \\
\text { BirUttam M. A. Rob Road } \\
\text { Azimpur Road (Up to Azimpur Bus Stand) }\end{array}$ \\
\hline 5 & Hatirjheel & Hatirjheel Link Road \\
\hline 6 & Rampura- Badda & $\begin{array}{l}\text { DIT Road } \\
\text { Banasree Main Road (upto Block C) } \\
\text { Bir Uttam Rafiqul Islam Avenue }\end{array}$ \\
\hline 7 & Baridhara- Badda & Progati Sarani Road (Up to Kuril Flyover) \\
\hline 8 & Gulshan- Mohakhali & $\begin{array}{l}\text { Gulshan Badda Link Road } \\
\text { Bir Uttam AK Khandakar Road } \\
\text { Shaheed Tajuddin Ahmed Avenue } \\
\text { Moghbazar Road (Up to Hatirjheel) }\end{array}$ \\
\hline 9 & Kamlapur & $\begin{array}{l}\text { Atish Diponkor Road (From Basabo to TT Para) } \\
\text { Kamlapur Road } \\
\text { Outer Circular Road } \\
\text { Kamlapur Road (From Kamlapur Railway Station to Arambagh) }\end{array}$ \\
\hline 10 & Fakirapul- Gulistan & $\begin{array}{l}\text { Toyenbee Circular Road } \\
\text { VIP Road } \\
\text { Shahid Syed Nazrul Islam Sharani } \\
\text { Bangabandhu Avenue (To Hazrat Golap Shah (Rh.) Mazar) }\end{array}$ \\
\hline
\end{tabular}




\section{Results and Discussion}

Inventory of plant species diversity

The presented study has been resulted in recording of total 90 plant species under 73 genera and 42 families from the road dividers of Dhaka city. For each species local name, scientific name, family, number individuals of each species (abundance), habit, origin status and usefulness are presented in Table 2. Plant species representation in the family is not equal. In this case $48 \%$ species represented by five families and $62 \%$ species represented by 37 families. The most dominant family is Caesalpiniaceae followed by Moraceae, Myrtaceae, Apocynaceae and Mimosaceae (Fig. 1).

The recorded plant species was categorized into different habit groups. Among them, 78\% species are represented by trees, $12 \%$ by shrubs, $7 \%$ by herbs and $3 \%$ by climbers (Fig. 2). Based on usefulness, the recorded plant species from road divider of Dhaka city categorized into several groups. Among the groups, ornamental plants category has the maximum plants followed by medicinal plants, wildlife supporting plants, timber yielding plants and fruits bearing plants respectively (Fig. 3).

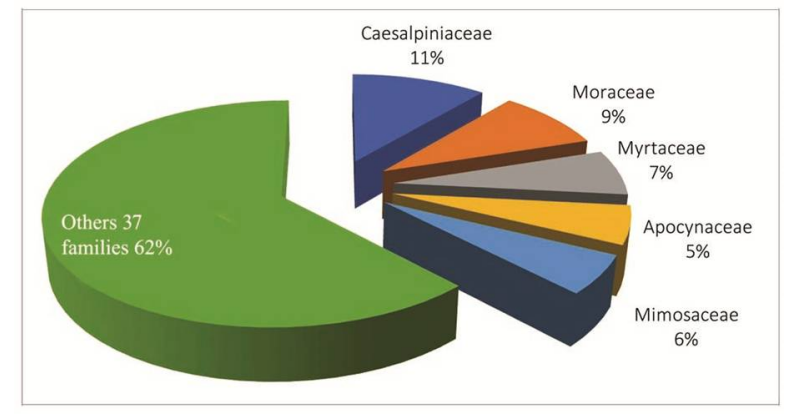

1

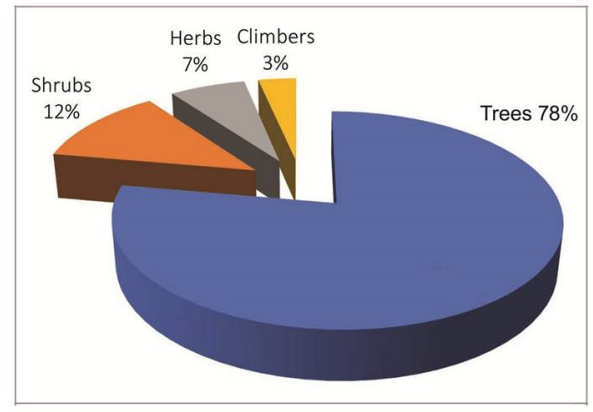

2

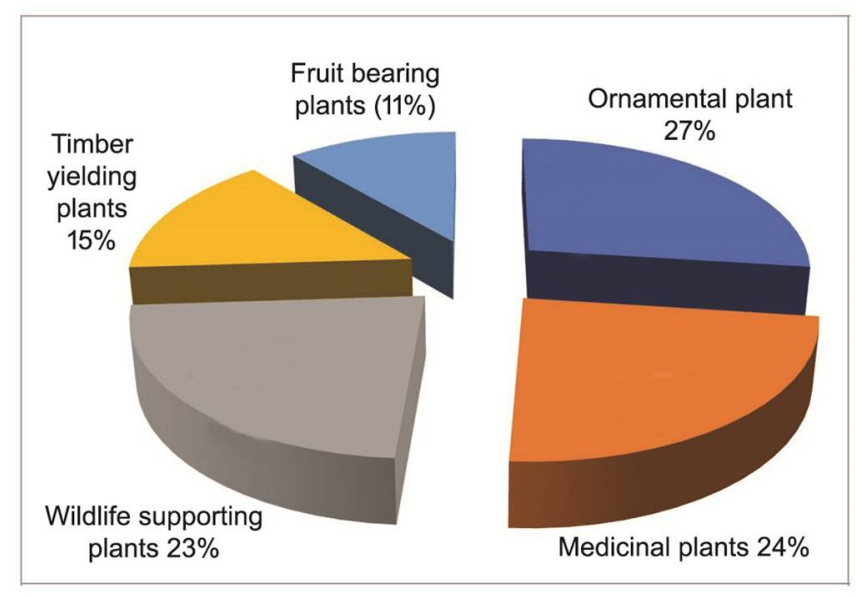

3

Figs 1-3: 1. Dominant families. 2. Plant species of different habit groups. 3. Plant species of different use groups. 
Table 2. Plant species diversity in the road dividers of Dhaka city.

\begin{tabular}{|c|c|c|c|c|c|c|c|}
\hline Scientific name & Local name & Family & $\begin{array}{l}\text { Presence in } \\
\text { site }\end{array}$ & $\begin{array}{l}\text { Abun- } \\
\text { dance }\end{array}$ & Habit & $\begin{array}{l}\text { Native or } \\
\text { Exotic }\end{array}$ & Uses \\
\hline $\begin{array}{l}\text { Accacia auriculiformis A. } \\
\text { Cunn. Ex Benth. And Hool. }\end{array}$ & Akashmoni & Mimosaceae & $1,2,4,6,9,10$ & 41 & $\mathrm{~T}$ & $\mathrm{E}$ & $\mathrm{T}$ \\
\hline Agave americana $\mathrm{L}$. & Century plant & Agavaceae & 5 & 3 & $\mathrm{~S}$ & $\mathrm{E}$ & $\mathrm{O}$ \\
\hline $\begin{array}{l}\text { Albizia procera (Roxb.) } \\
\text { Benth. }\end{array}$ & Shilkoroi & Mimosaceae & $4,9,10$ & 29 & $\mathrm{~T}$ & $\mathrm{~N}$ & $\mathrm{~T}, \mathrm{~W}$ \\
\hline $\begin{array}{l}\text { Albizia richardiana King } \\
\text { \&Prain }\end{array}$ & Gogon Sirish & Mimosaceae & $3,9,10$ & 6 & $\mathrm{~T}$ & $\mathrm{E}$ & $\mathrm{T}, \mathrm{W}$ \\
\hline Allamanda cathartica L. & Kolkeful & Apocynaceae & 5 & 4 & $\mathrm{C}$ & $\mathrm{E}$ & $\mathrm{M}, \mathrm{O}$ \\
\hline Alstonia scholaris (L.) R. Br. & Chatim & Apocynaceae & 3,10 & 3 & $\mathrm{~T}$ & $\mathrm{~N}$ & $\mathrm{M}, \mathrm{T}, \mathrm{W}$ \\
\hline Annona squamosa $\mathrm{L}$. & Ata & Annonaceae & 6 & 1 & $\mathrm{~T}$ & $\mathrm{E}$ & $\mathrm{F}, \mathrm{T}, \mathrm{W}$ \\
\hline $\begin{array}{l}\text { Araucaria heterophylla } \\
\text { (Salisb.) Franco }\end{array}$ & Chrismas tree & Araucariaceae & 5 & 3 & $\mathrm{~T}$ & $\mathrm{E}$ & $\mathrm{O}$ \\
\hline Artocarpus lakoocha Roxb. & Deoa & Moraceae & 6 & 1 & $\mathrm{~T}$ & $\mathrm{~N}$ & $\mathrm{~F}, \mathrm{~T}, \mathrm{~W}$ \\
\hline $\begin{array}{l}\text { Artocarpus heterophyllus } \\
\text { Lam. }\end{array}$ & Kanthal & Moraceae & $2,5,6,7$ & 7 & $\mathrm{~T}$ & $\mathrm{~N}$ & $\begin{array}{l}\mathrm{F}, \mathrm{T}, \mathrm{V}, \\
\mathrm{W}\end{array}$ \\
\hline Azadirachta indica A. Juss. & Neem & Meliaceae & $\begin{array}{l}1,2,3,4,5,6 \\
7,8,9,10\end{array}$ & 81 & $\mathrm{~T}$ & $\mathrm{~N}$ & $\mathrm{M}, \mathrm{T}, \mathrm{W}$ \\
\hline Bauhinia purpurea L. & Debkanchon & Caesalpiniaceae & 5,8 & 10 & $\mathrm{~T}$ & $\mathrm{~N}$ & $\mathrm{M}, \mathrm{O}$ \\
\hline Bauhinia variegata $\mathrm{L}$. & Roktokanchon & Caesalpiniaceae & 5 & 18 & $\mathrm{~T}$ & $\mathrm{E}$ & $\mathrm{M}, \mathrm{O}, \mathrm{T}$ \\
\hline $\begin{array}{l}\text { Bauhinia variegata var. } \\
\text { candida Voigt }\end{array}$ & $\begin{array}{l}\text { Shetrokto } \\
\text { kanchon }\end{array}$ & Caesalpiniaceae & 5 & 8 & $\mathrm{~T}$ & $\mathrm{E}$ & $\mathrm{M}, \mathrm{O}$ \\
\hline Bombax ceiba L. & Shimul & Bombacaceae & 5,6 & 2 & $\mathrm{~T}$ & $\mathrm{~N}$ & $\mathrm{O}, \mathrm{W}$ \\
\hline Borassus flabellifer L. & Taal & Arecaceae & $2,3,5,8,9,10$ & 20 & $\mathrm{~T}$ & $\mathrm{~N}$ & $\begin{array}{l}\mathrm{F}, \mathrm{M}, \mathrm{T}, \\
\mathrm{W}\end{array}$ \\
\hline $\begin{array}{l}\text { Bougainvillea spectabilis } \\
\text { Willd. }\end{array}$ & BaganBilash & Nyctaginaceae & $4,5,6,8,10$ & 73 & $\mathrm{C}$ & $\mathrm{E}$ & $\mathrm{O}$ \\
\hline $\begin{array}{l}\text { Butea monosperma (Lam.) } \\
\text { Taub. }\end{array}$ & Polash & Fabaceae & 5 & 1 & $\mathrm{~T}$ & $\mathrm{~N}$ & $\mathrm{O}, \mathrm{M}$ \\
\hline $\begin{array}{l}\text { Caesalpinia pulcherrima (L.) } \\
\text { Sw. }\end{array}$ & Radhachura & Caesalpiniaceae & 5 & 28 & $\mathrm{~S}$ & $\mathrm{E}$ & $\mathrm{O}, \mathrm{W}$ \\
\hline Callistemon citrinus Skeels & Bottle Brush & Myrtaceae & 4,5 & 12 & $\mathrm{~T}$ & $\mathrm{E}$ & $\mathrm{M}, \mathrm{O}$ \\
\hline Callistemon pallidus DC. & $\begin{array}{l}\text { Golden Bottle } \\
\text { Brush }\end{array}$ & Myrtaceae & 5 & 4 & $\mathrm{~T}$ & $\mathrm{E}$ & $\mathrm{M}, \mathrm{O}$ \\
\hline $\begin{array}{l}\text { Calotropis gigantea (L.) } \\
\text { Dryand. }\end{array}$ & Akondo & Asclepiadaceae & 5 & 1 & $\mathrm{~S}$ & $\mathrm{~N}$ & $\mathrm{M}, \mathrm{O}$ \\
\hline Canna indica $\mathrm{L}$. & Kolaboti & Cannaceae & 8 & 5 & $\mathrm{H}$ & $\mathrm{E}$ & $\mathrm{O}, \mathrm{M}$ \\
\hline Cassia fistula $\mathrm{L}$. & Sonalu & Caesalpiniaceae & $5,9,10$ & 5 & $\mathrm{~T}$ & $\mathrm{~N}$ & $\mathrm{M}, \mathrm{O}$ \\
\hline Cassia javanica $\mathrm{L}$. & Lalsonail & Caesalpiniaceae & 5 & 2 & $\mathrm{~T}$ & $\mathrm{E}$ & $\mathrm{O}$ \\
\hline Casuarina equisetifolia $\mathrm{L}$. & Jhau & Casuarinaceae & $2,5,8$ & 48 & $\mathrm{~T}$ & $\mathrm{E}$ & $\mathrm{O}$ \\
\hline $\begin{array}{l}\text { Catharanthus roseus (L.) } \\
\text { G.Don }\end{array}$ & Noyon Tara & Apocynaceae & 9 & 2 & $\mathrm{H}$ & $\mathrm{E}$ & $\mathrm{M}, \mathrm{O}$ \\
\hline $\begin{array}{l}\text { Citrus maxima (Burm.) } \\
\text { Merr. }\end{array}$ & Jambura & Rutaceae & 10 & 1 & $\mathrm{~T}$ & $\mathrm{E}$ & $\mathrm{F}, \mathrm{M}, \mathrm{W}$ \\
\hline $\begin{array}{l}\text { Clerodendrum inerme (L.) } \\
\text { Gaertn. }\end{array}$ & Bonjui & Verbenaceae & 5 & 23 & $\mathrm{C}$ & $\mathrm{N}$ & $\mathrm{O}, \mathrm{W}$ \\
\hline Crinum asiaticum $\mathrm{L}$. & Sukhdorshon & Amaryllidaceae & 8 & 1 & $\mathrm{H}$ & $\mathrm{N}$ & $\mathrm{O}, \mathrm{M}$ \\
\hline Cycas revolute Thunb. & Cycas & Cycadaceae & 5 & 8 & $\mathrm{~T}$ & $\mathrm{~N}$ & $\mathrm{O}$ \\
\hline
\end{tabular}


Table 2 contd.

\begin{tabular}{|c|c|c|c|c|c|c|c|}
\hline Scientific name & Local name & Family & $\begin{array}{l}\text { Presence in } \\
\text { site }\end{array}$ & $\begin{array}{l}\text { Abun- } \\
\text { dance }\end{array}$ & Habit & $\begin{array}{l}\text { Native or } \\
\text { Exotic }\end{array}$ & Uses \\
\hline Dalbergia sissoo DC. & Sishu & Fabaceae & 8 & 4 & $\mathrm{~T}$ & $\mathrm{E}$ & $\mathrm{T}$ \\
\hline Delonix regia (Hook.) Raf. & Krishnachura & Caesalpiniacae & $\begin{array}{l}1,2,4,5,8,9 \\
10\end{array}$ & 34 & $\mathrm{~T}$ & $\mathrm{E}$ & $\mathrm{O}, \mathrm{M}$ \\
\hline Diospyros blancoi A.DC. & Gaab & Ebenaceae & 4 & 2 & $\mathrm{~T}$ & $\mathrm{~N}$ & $\mathrm{~F}, \mathrm{~W}$ \\
\hline Dracaena spicata.Roxb. & Dracaena & Agavaceae & 9 & 1 & $\mathrm{~S}$ & $\mathrm{~N}$ & $\mathrm{O}$ \\
\hline Duranta erecta $\mathrm{L}$. & Duronto & Verbenaceae & 5 & 4 & S & $\mathrm{E}$ & $\mathrm{O}, \mathrm{M}$ \\
\hline $\begin{array}{l}\text { Dypsis lutescens (H.Wendl.) } \\
\text { Beentje\& J. Drans f. }\end{array}$ & Areca Palm & Arecaceae & 8 & 3 & $\mathrm{~T}$ & $\mathrm{E}$ & $\mathrm{O}, \mathrm{W}$ \\
\hline $\begin{array}{l}\text { Eucalyptus camaldulensis } \\
\text { Dehnhardt }\end{array}$ & Eucalyptus & Myrtaceae & $1,9,10$ & 19 & $\mathrm{~T}$ & $\mathrm{E}$ & $\mathrm{T}, \mathrm{M}$ \\
\hline Euphorbia tithymaloides L. & Rongchita & Euphorbiaceae & $5,6,8,9$ & 8 & $\mathrm{H}$ & $\mathrm{E}$ & $\mathrm{M}, \mathrm{O}$ \\
\hline Ficus benjamina L. & Jirbot & Moraceae & $1,2,4,5$ & 8 & $\mathrm{~T}$ & $\mathrm{~N}$ & W \\
\hline Ficus rumphii Blume & PakurBot & Moraceae & $4,5,6,8,9,10$ & 41 & $\mathrm{~T}$ & $\mathrm{~N}$ & $\mathrm{M}, \mathrm{T}, \mathrm{W}$ \\
\hline Ficus benghalensis L. & Lal Bot & Moraceae & $\begin{array}{l}1,2,3,4,6,7 \\
9,10\end{array}$ & 73 & $\mathrm{~T}$ & $\mathrm{~N}$ & $\mathrm{~T}, \mathrm{~W}$ \\
\hline $\begin{array}{l}\text { Ficus elastica Roxb. ex } \\
\text { Hornem. }\end{array}$ & Rubber Bot & Moraceae & $6,9,10$ & 5 & $\mathrm{~T}$ & $\mathrm{E}$ & $\mathrm{W}$ \\
\hline Ficus religiosa $\mathrm{L}$. & Ashwath Bot & Moraceae & $4,6,8,9,10$ & 22 & $\mathrm{~T}$ & $\mathrm{~N}$ & $\mathrm{~T}, \mathrm{~W}$ \\
\hline Grewia tenax (Forssk.) Fiori & Grewia & Malvaceae & 5 & 35 & S & $\mathrm{E}$ & $\mathrm{O}$ \\
\hline Ixora pavettaAndr. & Shetrongon & Rubiaceae & 8 & 9 & $\mathrm{~S}$ & $\mathrm{~N}$ & $\mathrm{O}, \mathrm{M}$ \\
\hline Ixora coccinea $\mathrm{L}$. & Rongon & Rubiaceae & $4,5,8,9,10$ & 42 & $\mathrm{~S}$ & $\mathrm{~N}$ & $\mathrm{O}$ \\
\hline $\begin{array}{l}\text { Khaya anthotheca (Welw.) } \\
\text { C. DC. }\end{array}$ & Lombu & Meliaceae & 10 & 1 & $\mathrm{~T}$ & $\mathrm{E}$ & $\mathrm{T}$ \\
\hline Lagerstroemia indica L. & Cherry & Lythraceae & $4,5,8$ & 37 & $\mathrm{~T}$ & $\mathrm{E}$ & $\mathrm{O}$ \\
\hline $\begin{array}{l}\text { Lagerstroemia speciosa (L.) } \\
\text { Pers. }\end{array}$ & Jarul & Lythraceae & 5,10 & 3 & $\mathrm{~T}$ & $\mathrm{~N}$ & $\mathrm{O}$ \\
\hline $\begin{array}{l}\text { Leucaena leucocephala (La } \\
\text { m.) de Wit }\end{array}$ & Epil-epil & Mimosaceae & $\begin{array}{l}2,3,4,5,6,7 \\
8,9,10\end{array}$ & 244 & $\mathrm{~T}$ & $\mathrm{E}$ & $\mathrm{T}$ \\
\hline Limonia acidissima Groff & Kodbel & Rutaceae & 6,9 & 2 & $\mathrm{~T}$ & $\mathrm{~N}$ & $\mathrm{~F}, \mathrm{M}, \mathrm{W}$ \\
\hline Litchi chinensis Sonn. & Lichu & Sapindaceae & 8 & 1 & $\mathrm{~T}$ & $\mathrm{E}$ & $\mathrm{F}, \mathrm{T}, \mathrm{W}$ \\
\hline $\begin{array}{l}\text { Litsea glutinosa (Lour.) } \\
\text { C.B.Rob. }\end{array}$ & Menda & Lauraceae & 1 & 1 & $\mathrm{~T}$ & $\mathrm{~N}$ & $\mathrm{M}, \mathrm{T}$ \\
\hline $\begin{array}{l}\text { Madhuca longifolia (J.Koeni } \\
\text { g ex L.) J.F.Macbr. }\end{array}$ & Mohua & Sapotaceae & 4 & 6 & $\mathrm{~T}$ & $\mathrm{~N}$ & $\mathrm{~F}, \mathrm{M}, \mathrm{W}$ \\
\hline Mangifera indica $\mathrm{L}$. & Aam & Anacardiaceae & $\begin{array}{l}1,2,4,5,6,8 \\
9,10\end{array}$ & 37 & $\mathrm{~T}$ & $\mathrm{~N}$ & $\begin{array}{l}\mathrm{F}, \mathrm{M}, \mathrm{T}, \\
\mathrm{W}\end{array}$ \\
\hline Melia azedarach L. & Gora Neem & Meliaceae & 4 & 2 & $\mathrm{~T}$ & $\mathrm{E}$ & $\mathrm{T}, \mathrm{W}$ \\
\hline Millettia peguensis Ali & Monihar & Fabaceae & 5 & 6 & $\mathrm{~T}$ & $\mathrm{E}$ & $\mathrm{O}$ \\
\hline Mimusops elengi L. & Bakul & Sapotaceae & $\begin{array}{l}2,3,4,5,6,7 \\
8,9,10\end{array}$ & 506 & $\mathrm{~T}$ & $\mathrm{E}$ & $\begin{array}{l}\text { M, O, T, } \\
\text { W }\end{array}$ \\
\hline Moringa oleifera Lam. & Sajina & Moringaceae & 4,8 & 2 & $\mathrm{~T}$ & $\mathrm{E}$ & $\begin{array}{l}\text { F, M, V, } \\
\text { W }\end{array}$ \\
\hline $\begin{array}{l}\text { Murraya paniculata }(\mathrm{L} .) \\
\text { Jack }\end{array}$ & Kamini & Rutaceae & $2,5,6,8,10$ & 26 & $\mathrm{~T}$ & $\mathrm{E}$ & $\mathrm{O}, \mathrm{M}$ \\
\hline Musa paradisiacal L. & Kola & Musaceae & 6,9 & 7 & $\mathrm{H}$ & $\mathrm{N}$ & $\begin{array}{l}\mathrm{F}, \mathrm{M}, \mathrm{V}, \\
\mathrm{W}\end{array}$ \\
\hline $\begin{array}{l}\text { Neolamarckia cadamba (Ro } \\
\mathrm{xb} \text {.) Bosser }\end{array}$ & Kadam & Rubiaceae & 2 & 3 & $\mathrm{~T}$ & $\mathrm{E}$ & $\begin{array}{l}\mathrm{M}, \mathrm{O}, \mathrm{V}, \\
\mathrm{W}\end{array}$ \\
\hline
\end{tabular}


Table 2 contd.

\begin{tabular}{|c|c|c|c|c|c|c|c|}
\hline Scientific name & Local name & Family & $\begin{array}{l}\text { Presence in } \\
\text { site }\end{array}$ & $\begin{array}{l}\text { Abun- } \\
\text { dance }\end{array}$ & Habit & $\begin{array}{l}\text { Native or } \\
\text { Exotic }\end{array}$ & Uses \\
\hline Nyctanthes arbor-tristis L. & Shiuli/Shefali & Oleaceae & 5 & 14 & $\mathrm{~S}$ & $\mathrm{E}$ & $\mathrm{M}, \mathrm{O}, \mathrm{W}$ \\
\hline $\begin{array}{l}\text { Peltophorum } \\
\text { pterocarpum (DC.) K. Heyne }\end{array}$ & Konokchura & Caesalpiniaceae & $5,6,8,10$ & 18 & $\mathrm{~T}$ & $\mathrm{E}$ & $\mathrm{O}, \mathrm{W}$ \\
\hline Phoenix sylvestris Roxb. & Khejur & Arecaceae & $5,6,7,8,9,10$ & 69 & $\mathrm{~T}$ & $\mathrm{~N}$ & $\mathrm{~F}, \mathrm{M}, \mathrm{W}$ \\
\hline $\begin{array}{l}\text { Phyllanthus acidus (L.) } \\
\text { Skeels }\end{array}$ & Orboroi & Euphorbiaceae & 6 & 6 & $\mathrm{~T}$ & $\mathrm{E}$ & $\mathrm{F}, \mathrm{M}, \mathrm{W}$ \\
\hline Plumeria alba $\mathrm{L}$. & Kath Golap & Apocynaceae & 5 & 1 & $\mathrm{~T}$ & $\mathrm{E}$ & $\mathrm{O}$ \\
\hline $\begin{array}{l}\text { Polyalthia longifolia (Sonn.) } \\
\text { Thwaites }\end{array}$ & Debdaru & Annonaceae & $\begin{array}{l}2,3,4,5,6,7 \\
8,9,10\end{array}$ & 456 & $\mathrm{~T}$ & $\mathrm{E}$ & $\mathrm{O}$ \\
\hline Psidium guajava $\mathrm{L}$. & Peyara & Myrtaceae & 6,8 & 3 & $\mathrm{~T}$ & $\mathrm{E}$ & $\begin{array}{l}\text { F, M, T, } \\
\text { W }\end{array}$ \\
\hline Putranjiva roxburghii Wall. & Putrojib & Euphorbiaceae & 4,8 & 21 & $\mathrm{~T}$ & $\mathrm{~N}$ & $\mathrm{M}, \mathrm{W}$ \\
\hline $\begin{array}{l}\text { Ravenala madagascariensis } \\
\text { Sonn. }\end{array}$ & Panthopadok & Strelitziaceae & 5 & 2 & $\mathrm{~T}$ & $\mathrm{E}$ & $\mathrm{O}$ \\
\hline $\begin{array}{l}\text { Roystonea regia (Kunth) } \\
\text { O.F. Cook }\end{array}$ & Royal Palm & Arecaceae & 5 & 20 & $\mathrm{~T}$ & E & $\mathrm{O}, \mathrm{T}$ \\
\hline $\begin{array}{l}\text { Samanea saman (Jacq.) } \\
\text { Merr.. }\end{array}$ & Raintree & Mimosaceae & 10 & 2 & $\mathrm{~T}$ & $\mathrm{E}$ & $\mathrm{T}$ \\
\hline $\begin{array}{l}\text { Senna polyphylla (Jacq.) } \\
\text { H.S. Irwin \& Barneby }\end{array}$ & Ranichura & Caesalpiniaceae & 5 & 15 & S & E & $\mathrm{O}$ \\
\hline Streblus asper Lour. & Sheora & Moraceae & 10 & 1 & $\mathrm{~T}$ & $\mathrm{~N}$ & $\mathrm{M}, \mathrm{W}$ \\
\hline $\begin{array}{l}\text { Swietenia mahagoni (L.) } \\
\text { Jacq. }\end{array}$ & Mahogani & Meliaceae & $\begin{array}{l}2,3,4,5,6,7 \\
8,9,10\end{array}$ & 125 & $\mathrm{~T}$ & $\mathrm{E}$ & $\mathrm{M}, \mathrm{T}, \mathrm{W}$ \\
\hline $\begin{array}{l}\text { Syngonium podophyllum } \\
\text { Schott }\end{array}$ & Podolotakochu & Araceae & 8 & 3 & $\mathrm{H}$ & $\mathrm{N}$ & $\mathrm{O}$ \\
\hline Syzygiumfruticosum DC. & Bhutijam & Myrtaceae & 5 & 1 & $\mathrm{~T}$ & $\mathrm{~N}$ & $\begin{array}{l}\mathrm{F}, \mathrm{M}, \mathrm{T} \\
\mathrm{W}\end{array}$ \\
\hline Syzygium cumini (L.) Skeels & Jam & Myrtaceae & $5,6,8,9,10$ & 16 & $\mathrm{~T}$ & $\mathrm{~N}$ & $\begin{array}{l}\mathrm{F}, \mathrm{M}, \mathrm{T}, \\
\mathrm{W}\end{array}$ \\
\hline $\begin{array}{l}\text { Tabernaemontana divaricata } \\
\text { (L.) Br. }\end{array}$ & Togor & Apocynaceae & $\begin{array}{l}3,4,5,6,7,8 \\
9,10\end{array}$ & 172 & S & $\mathrm{N}$ & $\mathrm{M}, \mathrm{O}$ \\
\hline Tamarindus indica $\mathrm{L}$. & Tetul & Caesalpiniaceae & $6,8,9,10$ & 12 & $\mathrm{~T}$ & $\mathrm{~N}$ & $\mathrm{~F}, \mathrm{M}, \mathrm{T}$ \\
\hline $\begin{array}{l}\text { Tecoma stans (L.) Juss. ex } \\
\text { Kunth }\end{array}$ & Haldeghonta & Bignoniaceae & 5,10 & 15 & $\mathrm{~T}$ & $\mathrm{E}$ & $\mathrm{O}$ \\
\hline Tectona grandis L.f. & Segun & Verbenaceae & $5,6,8$ & 3 & $\mathrm{~T}$ & $\mathrm{E}$ & $\mathrm{T}$ \\
\hline Terminalia catappa $\mathrm{L}$. & Kathbadam & Combretaceae & $1,4,5,8,9,10$ & 23 & $\mathrm{~T}$ & $\mathrm{E}$ & $\begin{array}{l}\mathrm{F}, \mathrm{M}, \mathrm{O} \\
\mathrm{W}\end{array}$ \\
\hline $\begin{array}{l}\text { Terminalia arjuna (Roxb. ex } \\
\text { DC.) Wight \&Arn. }\end{array}$ & Arjun & Combretaceae & $1,2,9,10$ & 17 & $\mathrm{~T}$ & $\mathrm{~N}$ & $\mathrm{M}, \mathrm{T}, \mathrm{W}$ \\
\hline $\begin{array}{l}\text { Terminalia bellirica (Gaertn. } \\
\text { ) Roxb. }\end{array}$ & Bohera & Combretaceae & $1,9,10$ & 5 & $\mathrm{~T}$ & $\mathrm{~N}$ & $\begin{array}{l}\text { F, M, T, } \\
\text { W }\end{array}$ \\
\hline Thuja orientalis L. & Thuja & Cupressaceae & $5,6,7,10$ & 50 & $\mathrm{~T}$ & $\mathrm{E}$ & $\mathrm{O}$ \\
\hline Trema orientalis (L.) Blume & Jibon & Ulmaceae & $4,8,9,10$ & 9 & $\mathrm{~T}$ & $\mathrm{~N}$ & $\mathrm{~W}$ \\
\hline Ziziphus mauritiana Lam. & Boroi & Rhamnaceae & $\begin{array}{l}1,5,6,7,8,9 \\
10\end{array}$ & 71 & $\mathrm{~T}$ & $\mathrm{~N}$ & $\mathrm{~F}, \mathrm{M}, \mathrm{W}$ \\
\hline
\end{tabular}

(Habit: $\mathrm{T}=$ Tree, $\mathrm{H}=\mathrm{Herb}, \mathrm{S}=$ Shrub, $\mathrm{C}=$ Climber) (Native or Exotic: $\mathrm{N}=$ Native, $\mathrm{E}=$ Exotic) $($ Uses: $\mathrm{T}=\mathrm{Timber}, \mathrm{M}=$ Medicinal, $\mathrm{F}=$ Fruit, $\mathrm{O}=$ Ornamental, $\mathrm{W}=$ Wildlife Supporting). 


\section{Dominant plant species}

One of our objectives is to determine the dominant plant species on the road dividers of Dhaka city. Plant species with their individual number were collected during the survey. Analysis showed that among the 90 species the most abundant plant species on the road dividers are Mimusops elengi (Bakul), Polyalthia longifolia (Debdaru), Leucaena leucocephala (Epil-epil), Tabernaemontana divaricata (Togor) and Swietenia mahagoni (Mahagoni, Table 3). The reason

Table 3.Top fifteendominant plant species with important value index.

\begin{tabular}{|c|c|c|c|c|c|}
\hline Scientific name & $\begin{array}{c}\text { Total } \\
\text { individuals }\end{array}$ & $\begin{array}{l}\text { Relative } \\
\text { density }\end{array}$ & $\begin{array}{l}\text { Relative } \\
\text { frequency }\end{array}$ & $\begin{array}{c}\text { Relative } \\
\text { abundance }\end{array}$ & $\begin{array}{l}\text { Importance } \\
\text { value index }\end{array}$ \\
\hline Mimusops elengi L. & 506 & 18.10376 & 14.25563 & 1.655634 & 34.01502 \\
\hline $\begin{array}{l}\text { Polyalthia longifolia (Sonn.) } \\
\text { Thwaites }\end{array}$ & 456 & 16.31485 & 11.79776 & 1.802875 & 29.91549 \\
\hline $\begin{array}{l}\text { Leucaena leucocephala (Lam.) } \\
\text { de Wit }\end{array}$ & 244 & 8.729875 & 8.286523 & 1.373466 & 18.38986 \\
\hline $\begin{array}{l}\text { Tabernaemontana divaricata } \\
\text { (L.) Br. }\end{array}$ & 172 & 6.153846 & 5.828656 & 1.37645 & 13.35895 \\
\hline Swietenia mahagoni (L.) Jacq. & 125 & 4.472272 & 4.213486 & 1.383785 & 10.06954 \\
\hline Azadirachta indica A.Juss. & 81 & 2.898032 & 4.283711 & 0.881993 & 8.063737 \\
\hline Ficus benghalensis L. & 73 & 2.611807 & 4.283711 & 0.794883 & 7.690401 \\
\hline Ziziphus mauritiana Lam. & 71 & 2.540251 & 4.073037 & 0.813093 & 7.426381 \\
\hline $\begin{array}{l}\text { Bougainvillea spectabilis } \\
\text { Willd. }\end{array}$ & 73 & 2.611807 & 2.317418 & 1.469328 & 6.398553 \\
\hline Phoenix sylvestris Roxb. & 69 & 2.468694 & 2.598317 & 1.238675 & 6.305686 \\
\hline Thuja orientalis L. & 50 & 1.788909 & 1.61517 & 1.44395 & 4.848029 \\
\hline Casuarina equisetifolia $\mathrm{L}$. & 48 & 1.717353 & 1.123596 & 1.992651 & 4.8336 \\
\hline Grewia tenax (Forssk.) Fiori & 35 & 1.252236 & 0.632023 & 2.583066 & 4.467325 \\
\hline Ficus rumphii Blume & 41 & 1.466905 & 1.825844 & 1.047419 & 4.340168 \\
\hline Ixora coccinea $\mathrm{L}$. & 42 & 1.502683 & 1.193821 & 1.641007 & 4.337511 \\
\hline
\end{tabular}

for the abundance of these tree species in the city is their aesthetic value or more availability of seedling of these trees in the nurseries or scarcity of native tree saplings or biodiversity promotion and native plants conservation issues might be ignored in some extend or plant taxonomist's knowledge was not taken much into account when planting. The widespread presence of these plant species in the city does not reflect the natural heritage of our country.

To determine the dominant tree species on road dividers of Dhaka city, Important Value Index was calculated. Results showed that by Mimusops elengi is the top dominant plant species followed Polyalthia longifolia, Leucaena leucocephala, Tabernaemontana divaricata and Swietenia mahagoni, respectively (Table 3). These plant species have also showed the higher relative density, relative frequency and relative abundance (Table 3). It is very unfortunate for us, among the 15 top dominant plant species 9 species are exotic and 6 are native. Common recorded native plant species in the road dividers are Azadirachta indica (Neem), Ficus benghalensis (Bot), Ficus rumphii (Pakur), Phoenix sylvestris (Khejur), Tabernaemontana divaricata (Togor, Table 3). 


\section{Exotic plant species}

Origin status of the recorded plants was tried to be explored in the present study. The result showed that $44 \%$ recorded plant species are native whereas $56 \%$ plant species are exotic (Fig.4). The higher number of exotic plant species is perhaps because of availability of sapling during plantation and might also be absence of plant taxonomic knowledge in species selection process during plantation. In most cases plantation program was conducted by contractor. Unfortunately, they have no such ability to differentiate between native and exotic plant species. Top dominant plant species on the road dividers are exotics including Mimusops elengi, Polyalthia longifolia, Leucaena leucocephala and Swietenia mahagoni (Table 4). They also showed higher abundance in the distribution on road dividers (Table 3). Possible origin of dominant exotic plant species are given in the Table 4. Apart from these other exotic tree species also found planted on the road dividers. Most common species are Acacia auriculiformis (Akashmoni), Dalbergia sissoo (Sishu), Delonix regia (Krichnachura), Eucalyptus camaldulensis (Eucalyptus), Peltophorum pterocarpum (Konokchura) and Terminalia catappa (Katbadam). These all are soft wooded trees, prone to break down easily. These plant species may create huge hazard in densely populated city of Dhaka during natural disasters and most cases not so friendly with animal diversity though some has aesthetic value. Their branching patterns of roots, stems and canopy are not road divider friendly. Tree falls occurred in every year during torrential rain with gusty wind and nor'ester which caused huge cost. So, the authority responsible for plantations in the islands and didn't give enough thought on conserving and planting native plants of Bangladesh whereas this city could have been an epitome for displaying the native floral culture, supporting the native birds and satisfying the aesthetic needs of its dwellers, the road dividers have been a failure to some extent.

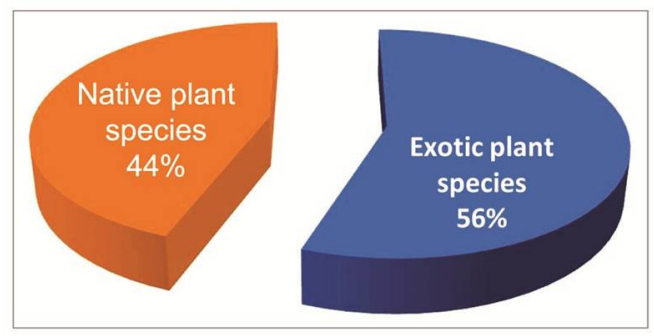

Fig. 4. Origin status of plant species.

Table 4. Dominant exotic tree species and their origin.

\begin{tabular}{lll}
\hline Local name & Scientific name & $\begin{array}{l}\text { Origin (Pasha and Hossain, 2004 and Akter and Zuberi, } \\
\text { 2009). }\end{array}$ \\
\hline Bakul & Mimusops elengi L. & $\begin{array}{l}\text { Native to India, Sri Lanka, the Andaman Islands, Myanmar } \\
\text { and Indo-China }\end{array}$ \\
Debdaru & $\begin{array}{l}\text { Polyalthia longifolia } \text { (Sonn.) } \\
\text { Thwaites }\end{array}$ & $\begin{array}{l}\text { Native to southern India and Sri Lanka } \\
\text { Epil-epil }\end{array}$ \\
$\begin{array}{l}\text { Leucaena leucocephala (La } \\
\text { m.) de Wit }\end{array}$ & Native to southern Mexico and northern Central America \\
Mahagoni & $\begin{array}{l}\text { Swietenia mahagoni (L.) } \\
\text { Jacq. }\end{array}$ & $\begin{array}{l}\text { Native to South Florida in the United States and islands in } \\
\text { the Caribbean including the Bahamas, Cuba, Jamaica, and } \\
\text { Hispaniola. }\end{array}$ \\
\hline
\end{tabular}


Plant species diversity

The diversity measure (expressed by $\mathrm{H}$ ) was primarily developed within information theory (Shannon, 1948) but later the measure was adopted in studies on species diversity (Margalef, 1957). In principle, Shannon's H takes into account the proportion of each species in an ecosystem studied; hence, it gives a better description of an ecosystem's diversity than a plain number of species (Konopiński 2020). When the number of species is equal in two locations, the index is capable of distinguishing between sites dominated by a single or only a few predominant species and those where each species has comparable input to the whole biodiversity (Margalef, 1957).Plant species diversity measured by Shannon Index (H) values were varied from 3.17 to 1.66 (Table 5). The top $\mathrm{H}$ value was found in Hatirjeel site because the area was recently planted by multiple plant species with more or less equal number of individuals. The $\mathrm{H}$ value was found lowest in the Baridhara-Dadda and Hatirpul-Panthapath because the area was planted by few species with unequal number of individuals. That means particular species was given high priority during plantation time. The other sites the $\mathrm{H}$ values were found more than two. That means in these sites also planted by many plant species were planted with nearly equal number of individuals.

Table 5. Comparative Shannon-Weiner Diversity Index values among the study sites.

\begin{tabular}{lccc}
\hline Site name & $\begin{array}{c}\text { Number of } \\
\text { species }\end{array}$ & $\begin{array}{c}\text { Number of } \\
\text { Individuals }\end{array}$ & $\begin{array}{c}\text { Shannon-Weiner } \\
\text { Diversity Index }\end{array}$ \\
\hline Hatirjheel & 54 & 819 & 3.17 \\
Rampura-Badda & 30 & 220 & 2.73 \\
Kamlapur & 37 & 322 & 2.71 \\
Fakirapul, & 37 & 299 & 2.61 \\
Gulshan-Mohakhali & 34 & 487 & 2.55 \\
Dhanmondi-, Parliament & 26 & 221 & 2.43 \\
Area-Azimpur & & & \\
Mirpur & 19 & 215 & 2.26 \\
Uttara & 12 & 49 & 2.12 \\
Hatirpul-Panthapath & 9 & 61 & 1.85 \\
Baridhara-Badda & 10 & 89 & 1.66 \\
\hline
\end{tabular}

\section{Recommendations}

Total 90 species of plants have been recorded from the road dividers of Dhaka city. This is not uncommon in terms of species diversity but the problem is elsewhere. Substantial differences in population numbers can be observed under these species. With a population of few species including Mimusops elengi, Polyalthia longifolia, Leucaena leucocephala and Swietenia mahagoni, the whole city is being occupied which is at all not conducive to environment, biodiversity and beautification of Dhaka city. Moreover, among the abundant, dominant, and common species, all plants are exotics except Tabernaemontana divaricata plant. Due to the high presence of all these exotic tree species, our native plant species are getting lost day by day. With this loss, our native nature is losing its heritage. Even animals, birds, butter flies, honey bees, insect and other pollinators those depend on native plant species are getting lost. The new generations of students are being deprived of opportunity to get acquainted with our native species of flora and fauna. We have many indigenous ornamental plant species that are endangered in their natural habitats today. Given the importance of planting these species to enhance the beauty of the 
city, on the one hand, there is a possibility that the species will survive in our country, on the other hand our natural heritage will be protected. We want our favorite city of Dhaka to be adorned with ornamental plant of native species. However, with the interest of environment, ecology, biodiversity and beautification, the current status of plant species diversity on the road dividers needs to be rethought.

A number of recommendations are made based on the present results for the management of plant diversity on the road dividers of Dhaka city. First recommendation is to make a master plan for the beautification of Dhaka city using native plant species. In this case local stakeholders including city corporations, parks and gardens authorities, RAJUK, forest department and experts should be brought under one umbrella. Second, immediate management actions should be taken for those exotic species already planted. Existing exotic plant species including Mimusops elengi, Polyalthia longifolia, Leucaena leucocephala, Swietenia mahagoni,Samanea saman, Acacia auriculiformis, Dalbergia sissoo, Delonix regia, Eucalyptus camaldulensis, Peltophorum pterocarpum and Terminalia catappa should be taken under management plan. If necessary, cutting, trimming and dressing can be done. Gradually exotic replacement can be done by planting native multipurpose plant species. Third, for the augmentation of wildlife and biodiversity, native, multipurpose and local plant species of Dhaka should be given priority for the future plantation programs in this mega city. City people want to acquaint with local environment and natural heritage of native plants and animals. In case of wide road dividers with high soil depth, medium sized native trees with wildlife supporting characters can be planted and those plants must be under regular management program, e.g. Butea monosperma (Polash), Bombax ceiba (Shimul), Shorea robusta (Sal), Miliusa velutina (Gandhi gazari) Alstonia scholaris (Chatim), Sterculia villosa (Udal), Neolamarckia cadamba (Kadam), Azadirachta indica (Neem), Terminalia chebula (Horitaki), Terminalia bellirica (Bohera), Terminalia arjuna (Arjun), Syzygium cumini (Jam), Phyllanthus emblica (Amloki), Tamarindus indica (Tentul), Diospyros malabarica (Deshi gab), Toona ciliata (Toon), Litsea glutinosa (Menda), Ficus racemosa (Jogdumur), Crateva nurvala (Borun), could be selected for the plantation (Datta and Mitra,1953). Fourth, if road dividers are narrow and low soil depth, priority should be given on native low height shrubby plant species, e.g. Holarrhena pubescens (Kurchi), Grewia microcos (Aser), Tabarnaemontana divericata (Togor) and Ixora coccinea (Rongon) with different periods of flowering to fulfill the aesthetic need and also small plant species, namely, Melastoma malabathricum (Datranga), Woodfordia fruticosa (Dhaiful) and Clerodendrum viscosum (Bhat) could be planted (Datta and Mitra 1953). Fifth, for aesthetic reason, each and every different road dividers could be ornamented by different native plant species of different flowering seasons. For example, Dhanmondi road divider could be ornamented by Cassia fistula (Sonalu), Manik Mia Avenue by Butea monosperma (Polash) and Bombax ceiba (Shimul). Sixth, nurseries should be established under forest department to meet the challenge in finding saplings of native plant species. Seventh, opinion of plant taxonomists should be taken in the selection process of plant species and plantation sites selection.

\section{Conclusions}

The results of present evaluation of plant species diversity recorded from the road dividers of Dhaka city provides a basis for future management plan. The record of 90 plant species under 42 families on the road dividers is the indication of richness in floral diversity. Unfortunately, few exotic plant species contributed maximum individuals. This is alarming in future for the environment of Dhaka city to sustain biodiversity. The most abundant species are Mimusops elengi, Polyalthia longifolia, Leucaena leucocephala and Swietenia mahagoni. Apart from these, 
Acacia auriculiformis, Dalbergia sissoo, Delonix regia, Eucalyptus camaldulensis, Peltophorum pterocarpum and Terminalia catappa are observed on the road dividers. Mimusops elengi is the top dominant plant species followed by Polyalthialongifolia, Leucaena leucocephala, Tabernaemontana divaricata and Swietenia mahagoni. These plant species have also showed the higher relative density, relative frequency and relative abundance in Dhaka city. The percentage of exotic plant species was higher than native plant species. Most abundant and dominant plant species on the road dividers are also exotics including Mimusops elengi, Polyalthia longifolia, Leucaena leucocephala and Swietenia mahagoni. Abundance and dominance data of plant species in road dividers may assist concerned stakeholders in their aims to increase native species and reduce exotic plant species. Species diversity value was found higher in Hatirjeel site and lowest in Baridhara-Badda site. Based on the results of present evaluation of plant species diversity, a number of recommendations were made for the better management plant diversity on the road dividers to fulfill both aesthetic needs and biodiversity conservation in Dhaka city. Some recommended native plant species for plantation according to the width and soil depth in the road dividers are Cassia fistula, Holarrhena pubescens, Shorea robusta, Miliusa valutina, Butea monosperma, Alstonia scholaris, Toona ciliata, Ficus racemosa, Bombax ceiba, Woodfordia fruticosa, Melastoma malabathricum, Sterculia villosa, Grewia microcos, Syzygium cumini, Tabernaemontana divaricata, Ixora coccinea, Terminalia bellirica, and Terminalia arjuna and also native seasonal flowers.

\section{Acknowledgement}

The authors are thankful to the University Grants Commission (UGC) and University of Dhaka for providing financial support to conduct this research work.

\section{References}

Ahmed, Z.U., Begum, Z.N.T., Hassan, M.A., Khondker, M., Kabir, S.M.H., Ahmad, M., Ahmed, A.T.A., Rahman, A.K.A. and Haque, E.U. (Eds) 2008a.Encyclopedia of Flora and Fauna of Bangladesh, Vol. 6. Angiosperms: Dicotyledons (Acanthaceae - Asteraceae). Asiatic Society of Bangladesh, Dhaka, pp. 1408.

Ahmed, Z.U., Hassan, M.A., Begum, Z.N.T., Khondker, M., Kabir, S.M.H., Ahmad, M., Ahmed, A.T.A., Rahman, A.K.A. and Haque, E.U. (Eds) 2008b.Encyclopedia of Flora and Fauna of Bangladesh, Vol. 12. Angiosperms: Monocotyledons (Orchidaceae - Zingiberaceae). Asiatic Society of Bangladesh, Dhaka, pp. 1-552.

Ahmed, Z.U., Hassan, M.A., Begum, Z.N.T., Khondker, M., Kabir, S.M.H., Ahmad, M., Ahmed, A.T.A., Rahman, A.K.A. and Haque, E.U. (Eds) 2009a.Encyclopedia of Flora and Fauna of Bangladesh, Vol. 7. Angiosperms: Dicotyledons (Balsaminaceae - Euphorbiaceae). Asiatic Society of Bangladesh, Dhaka, pp. 1-546.

Ahmed, Z.U., Hassan, M.A., Begum, Z.N.T., Khondker, M., Kabir, S.M.H., Ahmad, M., Ahmed, A.T.A., Rahman, A.K.A. and Haque, E.U. (Eds) 2009b.Encyclopedia of Flora and Fauna of Bangladesh, Vol. 8. Angiosperms: Dicotyledons (Fabaceae - Lythraceae). Asiatic Society of Bangladesh, Dhaka, pp. 1-478.

Ahmed, Z.U., Hassan, M.A., Begum, Z.N.T., Khondker, M., Kabir, S.M.H., Ahmad, M. and Ahmed, A.T.A. (Eds) 2009c.Encyclopedia of Flora and Fauna of Bangladesh, Vol. 9. Angiosperms: Dicotyledons (Magnoliaceae - Punicaceae). Asiatic Society of Bangladesh, Dhaka, pp. 1-488.

Ahmed, Z.U., Hassan, M.A., Begum, Z.N.T., Khondker, M., Kabir, S.M.H., Ahmad, M., and Ahmed, A.T.A. (Eds) 2009d.Encyclopedia of Flora and Fauna of Bangladesh, Vol. 10. Angiosperms: Dicotyledons (Ranunculaceae - Zygophyllaceae). Asiatic Society of Bangladesh, Dhaka, pp. 1-580.

Akter, A. and Zuberi, M.I. 2009. Invasive alien species in Northern Bangladesh: identification, inventory and impacts. International journal of biodiversity and conservation 1(5): 129-134. 
Alam, M.K. 1967. Palms in Dhaka City. M. Sc. thesis, University of Dhaka, Dhaka.

Aronson, M.F., Lepczyk, C.A., Evans, K.L., Goddard, M.A., Lerman, S.B., MacIvor, J.S., and Vargo, T. 2017. Biodiversity in the city: key challenges for urban green space management. Frontiers in Ecology and the Environment 15(4): 189-196.

Banglapedia. 2014: The National Encyclopedia of Bangladesh. Asiatic Society Dhaka, Bangladesh.

Cronquist, A. 1981.An integrated system of classification of flowering plants.Columbia University Press, New York.pp. 1262.

Datta, R.M. and Mitra, J.N. 1953. Common plants in and around Dacca. Bull. Bot. Soc. Beng. 7(1-2): 1-110.

Dewan, A.M. and Yamaguchi, Y. 2009. Land use and land cover change in Greater Dhaka, Bangladesh: Using remote sensing to promote sustainable urbanization. Applied geography 29(3): 390-401.

Goldsmith, F.B. and Harrison, C.M. 1976. Description and Analysis of Vegetation. In: Chapman, S.B., Ed., Methods in Plant Ecology, John Wiley and Sons, New York, 85-155.

Hossain, A.B.M.E. and Uddin M.Z. 2011.Plants of Modern Period (from 1947 to the present time).In: Celebration of 400 years of Dhaka. pp. 63-82.

Hossain, A.B.M.E. 1966. Compositae of Dhaka city and it's suburbs. M. Sc. thesis, University of Dhaka, Dhaka.

Hossain, A.B.M.E. 2006. Dhaka Metropolis: Urban Greening and Green Spaces. In: People's report 20042005 (eds. A. Rahman and M. Hossain).

Hough, M. 2004. Cities and Natural Process. Rutledge: London, UK, pp. 64-65. ISBN 0-415-29855-5.

Huq, A.M. and Begum, M. 1984.An annotated list of climbers of Dacca.Bangladesh National Herbarium, BARC, Dhaka, pp.1-7.

Hussain, G. 1965. Papilionaceae of Dhaka city and it's suburbs. M. Sc. Thesis, Dhaka University, Dhaka.

Khan, M.S. and Huq, A.M. 1981. An annotated List of Trees of Dhaka. Privately published by Authors.

Konopinski, M.K. 2020. Shannon diversity index: a call to replace the original Shannon's formula with unbiased estimator in the population genetics studies. PeerJ 8:e9391 https://doi.org/10.7717/peerj.9391.

Krebs, C.J. 1989. Ecological Methodology.Harper Collins Publishers. New York, 654pp.

Marco, A., Dutoit, T., Deschamps-Cottin, M., Mauffrey, J.F., Vennetier, M. and Bertaudière-Montes, V. 2008. Gardens in urbanizing rural areas reveal an unexpected floral diversity related to housing density. C.R. Biol. 331: 452-465.

Margalef, R. 1957. La teoría de la información en Ecología. Memorias de la Real Academia de Cienciasy Artes de Barcelona, 32:373-436.

McKinney, M.L. 2008. Effects of urbanization on species richness: a review of plants and animals. Urban ecosystems 11(2): 161-176.

Pasha, M.K., and Hossain, M.K. 2004. An account of the exotic flora of Bangladesh. Journal of Forestry and Environment 2: 99-115.

Pyšek, P. 1998. Alien and native species in Central European urban floras: a quantitative comparison. Journal of Biogeography 25(1): 155-163.

Pyšek, P.and Richardson, D.M. 2010.Invasive species, environmental change and management, and health. Annual review of environment and resources 35: 25-55.

Rahman, M. 1966. Caesalpinioideae of Dhaka City and it's Suburbs. M. Sc. Thesis, Dhaka University, Dhaka.

Rahman, S., Hasan, S.M.R., Islam, M.A. and Maitra, M.K. 2011.Temporal change detection of vegetation coverage of Dhaka using remote sensing. International Journal of Geomatics and Geosciences 2(2):481490.

Sayed, A., Salam, M.Z.E.I., Alam, M.R.B. and Khan, N.N. 2015.A Study of Ground Surface Motion for Different Locations of Dhaka City. DOI:10.9790/1684-12616171.

Shannon, C.E. 1948. A mathematical theory of communication. Bell System Technical Journal 27:379-423. 
Siddiqui, K.U., Islam, M.A., Ahmed, Z.U., Begum, Z.N.T., Hassan, M.A., Khondker, M., Rahman, M.M., Kabir, S.M.H., Ahmad, M., Ahmed, A.T.A., Rahman, A.K.A. and Haque, E.U. (Eds) 2007c.Encyclopedia of Flora and Fauna of Bangladesh, Vol. 11. Angiosperms: Monocotyledons (Agavaceae -Najadaceae). Asiatic Society of Bangladesh, Dhaka, pp. 1-399.

Singh, A.K., Singh, H and Singh J.S. 2018. Plant Diversity in Cities: Call for Assessment and Conservation. Current Science 115 (15): 428-435.

Smith, R.M., Gaston, K.J., Warren, P.H. and Thompson, K. 2005. Urban domestic gardens: relationships between landcover composition, housing and landscape. Landscape Ecol. 20(2): 235-253.

Sukopp, H. 1990. Urban ecology and its application in Europe.Urban ecology. pp. 1-22.

The Daily Star 2016. Artist Mithu dies as tree falls on his rickshaw. <https://www.thedailystar.net/ backpage/artist-mithu-dies-tree-falls-his-rickshaw-788005>. The Daily Star, Dhaka, Bangladesh. Retrieved on 11 May 2021.

The Daily Star 2017. Man dies as tree falls on bike in Dhaka. <https://www.thedailystar.net/city/man-killedtree-falls-bike-1378267>. The Daily Star, Dhaka, Bangladesh.Retrieved on 11 May 2021.

The Daily Star 2018. Man dies as tree falls on him in Dhanmondi Lake. <https://www.thedailystar. net/country/man-dies-tree-falls-him-dhanmondi-lake-1574914>.The Daily Star, Dhaka, Bangladesh. Retrieved on 11 May 2021.

Uddin, M.Z. and Hassan, M.A. 2016. Plant diversity of Dhaka University campus, Bangladesh. Journal of Asiatic Society 42(1): 49-68.

Uddin, M.Z., Mitu ,F.Y., Rifat, A.B. and Kaium, A.A. 2019. Ethnomedicinal study focusing on anti-diabetic plants used by the community living in and around Dhaka. Bangladesh J. Plant Taxon. 26(2): 231-247.

Zeauddin, M. 1967. Angiospermic Climbers of Dhaka City and it's Suburbs. M. Sc. Thesis, Dhaka University, Dhaka.

(Manuscript received on 02 December, 2020; revised on 20 May, 2021) 